\title{
Prevalence of Osteoporosis and Risk of Developing Fragility Fracture in Post-Menopausal Women
}

\author{
Muhammad Hafiz Bin Hamzah ${ }^{1}$, Aminudin Che Ahmad ${ }^{1}$ \\ ${ }^{1}$ Department of Orthopaedics, Traumatology and Rehabilitation, Kulliyyah of \\ Medicine, International Islamic University Malaysia
}

Presenter: Muhammad Hafiz Bin Hamzah

Introduction: Osteoporosis and fragility fracture pose major health concern and cause significant financial burdens in healthcare system. The objectives of this study were to determine the prevalence of osteoporosis among post-menopausal women and to predict the risk of having the major osteoporotic and hip fracture in 10 years using the WHO Fracture Risk Assessment Tool. Materials and Methods: This was a cross-sectional study spanning over a two-year period (from June 2015 to June 2017) investigating post-menopausal women who did their Bone Mass Density (BMD) test at Hospital Melaka. Collected BMD data and parameters were used for analysif of the prevalence of osteoporosis among the study population and FRAX calculation was used to predict the average risk of fragility fracture in 10 years. Statistical analysis was done using SPSS statistical package. Results: Prevalence of osteoporosis among 388 post-menopausal women age 45 to 90 years old was $29.9 \%$. More than half of patients had osteoporosis were Chinese $(51 \%)$ followed by Malay (43\%), Indian (5\%) and others (1\%). The 10 years average percentage of developing major osteoporosis was $10.81 \pm 9.55 \%$, while the average percentage of developing hip fracture was $4.20 \pm 6.53$. Conclusion: Prevalence of the post-menopausal women who were osteoporotic was about $30 \%$ which is comparable to other countries (Japan, Egypt, Australia, Mexico, Argentina and India). The 10 years average percentage of developing major osteoporosis and hip fracture in our study is quite high, approximately around $20 \%$ and $10 \%$, respectively. 